\title{
Inovação Responsável no Âmbito da Gestão e Negócios
}

Luciana Maines da Silva (UNISINOS) - lucmasi@gmail.com

\begin{abstract}
RESUMO: Essa pesquisa teve por objetivo analisar a produção científica sobre a aplicação do conceito de inovação responsável voltada à área de gestão e negócios. Através de um estudo bibliométrico, foram identificados trinta e três artigos internacionais e um nacional. A análise de conteúdo apontou que a maioria dos estudos são voltados para a governança da inovação e sua relação com políticas públicas. Outros estudos abordaram o processo de desenvolvimento da inovação responsável, o âmbito da inovação, a relação com os stakeholders e ética. As lentes teóricas utilizadas nos estudos se voltaram para governança, recursos, custos de transação e responsabilidade social corporativa. As pesquisas aplicadas limitam-se as áreas de saúde e alimentos, adoção de tecnologia verde, pequenas empresas, sistemas de informação, superinteligência e nanotecnologia. Se sugere, por fim, a realização de pesquisas utilizando outras lentes teóricas, como a Teoria Institucional e Capacidades Dinâmicas, assim como avaliar de que forma a universidade influencia a responsabilidade na inovação daquelas empresas com que se relaciona.
\end{abstract}

PALAVRAS-CHAVE: Pesquisa e Inovação Responsáveis; Inovação Responsável; Inovação, Estudo bibliométrico.

\section{Responsible Innovation in Management and Business}

ABSTRACT: This research analyzed the management and business academic production focused on the concept of responsible innovation. Using a bibliometric method, thirty-three international articles and one national were identified. Content analysis pointed out that most of the studies are focused on innovation governance and its relationship with public policies. Other studies addressed the process of developing responsible innovation, the innovation's scope, the relationship with stakeholders and ethics. Governance, resources, transaction costs and corporate social responsibility are the theories used. The empirical research is limited to health and food, adoption of green technology, small businesses, information systems, superintelligence and nanotechnology. Finally, it is suggested the use of other theories, such as the Institutional Theory and Dynamic Capabilities, as the evaluation of universities influence on companies' responsible innovation process.

KEYWORDS: Research and Innovation Responsible; Responsible Innovation; Innovation, Bibliometric Research.

\section{Universidade \\ U1m Federal \\ Fluminense}

R. Desembargador Ellis Hermydio Figueira, 783, Bloco A, sl. 304, Aterrado. 27213-145 - Volta Redonda, RJ - Brasil

www.uff.br

Copyright (C) 2018 RASI. Todos os direitos, até mesmo de tradução, são reservados. É permitido citar parte de artigos sem autorização prévia, desde que seja identificada a fonte 


\section{Inovação Responsável no Âmbito da Gestão e Negócios}

\section{Introdução}

O século XX foi responsável pelos maiores avanços tecnológicos da humanidade tais como motores à combustão interna, energia nuclear, biotecnologia e a revolução da tecnologia da informação. Mas foi no início do século XXI que seus efeitos foram percebidos. Todas essas inovações geraram crescimento econômico, mas também trouxeram consequências negativas como mudanças climáticas e crises econômicas globais, através da criação de complexos instrumentos financeiros, que tiveram forte impacto negativo na economia (Owen, Maynard, \& Depledge, 2009).

Um dos primeiros estudos sobre o impacto da inovação foi feito por Duke (1978). O objetivo era o de analisar a participação de um corpo de professores (inclusive sua própria) nas três fases de projetos de pesquisa e desenvolvimento (planejamento, implementação e avaliação) visando melhorar o desempenho de estudantes de áreas menos favorecidas. Mas foi a partir dos anos 2000 que foram desenvolvidos a maioria dos estudos voltados a avaliar o impacto das inovações.

O termo inovação responsável (IR) surgiu em 2002, no 6th EU Framework Programme for Research and Technological Development (FP6), que é um conjunto de ações no nível da União Europeia para financiar e promover a pesquisa (Comissão Europeia, 2002). No documento o termo está atrelado a questões éticas (criação de redes entre organismos e atividades existentes, promoção do diálogo em um contexto global, conscientização, treinamento, pesquisa em ética em relação à ciência e tecnologia) e a incerteza, risco e princípio da precaução (análise e melhores práticas). Contudo, processos e ferramentas já eram utilizados antes disso, a exemplo do filósofo francês Michel Serres, que, por volta de 1972, convidava outros filósofos a trabalhar juntamente com cientistas e engenheiros no desenvolvimento técnico-científico (Burget, Bardone, \& Pedaste, 2016). Ao longo da última década, o conceito de inovação impulsionou grande parte da agenda de pesquisa europeia (Comissão Europeia, 2013).

A responsabilidade na pesquisa e inovação é motivada pela preocupação global com o planeta, seus recursos naturais, assim como uma sociedade justa e inclusiva (Nações Unidas, 2015). Essa preocupação diz respeito a governos, que podem agir através de políticas e regulamentos, mesmo que ainda estejam em fase de desenvolvimento (Scherer, Palazzo, \& Baumann, 2006). Mas também diz respeito à iniciativa privada, alinhando as demandas de pesquisa e desenvolvimento (P\&D) com valores, necessidades e expectativas da sociedade (Burget et al., 2016).

Muitos estudos sobre o tema estão voltados à nanotecnologia, que surgiu como uma promessa no aumento da produtividade, melhor desempenho e novas funcionalidades (Robinson, 2009) assim como redução no uso de substâncias químicas, consumo de energia e recursos e geração de resíduos. Isso tem potencializado o desenvolvimento de novos produtos e aplicações em vários setores industriais e de consumo, como o de saúde, cosméticos, tintas e revestimentos, eletrônicos, alimentos e embalagens alimentares. Apesar dos aparentes benefícios, a preocupação se dá na exposição dos trabalhadores durante a produção, utilização ou reciclagem de nanoprodutos industriais assim como os consumidores durante a utilização de produtos de consumo. A preocupação também se dá com o meio ambiente, uma vez que os nanoprodutos muitas vezes são compostos por prata, óxido de alumínio e sílica (Som, Berges, Chaudhry, Dusinska, Fernandes, Olsen, \& Nowack, 2010). Por outro lado, a nanotecnologia 
pode contribuir positivamente para o meio ambiente, através do aumento da eficiência da captura de carbono de fontes fixas (como as centrais elétricas), assim como a captura de $\mathrm{CO} 2$ da atmosfera, por exemplo, usando fotoreatores domésticos distribuídos, o que também pode converter carbono capturado em produtos como combustíveis (Owen, \& Goldberg, 2010).

Outros estudos estão voltados à área da biotecnologia, como os biorepositórios e biobancos (Gaskell, Gottweis, Starkbaum, Gerber, Broerse, Gottweis, ... \& Soulier, 2013) e sobre reprodução humana (Dondorp, \& de Wert, 2011), onde a discussão recai sobre questões éticas, incluindo formas de consentimento, privacidade e proteção de dados (Gaskell, Gottweis, Starkbaum, Gerber, Broerse, Gottweis, ... \& Soulier, 2013). Há, ainda, estudos voltados ao meio ambiente - como o impacto do uso de aerossóis (Pidgeon, Parkhill, Corner, \& Vaughan, 2013) - e socioambientais - criação de cabras em países em desenvolvimento (Escareño, Salinas-González, Wurzinger, Iñiguez, Sölkner, \& Meza-Herrera, 2012). Como se pode perceber, a maioria dos estudos são voltados à aspectos ambientais e éticos assim como voltados à área da saúde. Mais recentemente o tema se tornou foco de estudos na área de gestão e negócios, pela influência sobre as organizações. Assim, considerando a importância do tema, o objetivo desse artigo é o de analisar a aplicação do conceito de inovação responsável voltada à área de gestão e negócios.

O artigo está estruturado em seções que abordam o conceito de inovação responsável, a revisão da literatura dos estudos sobre pesquisa e inovação responsável; a metodologia aplicada; a análise dos resultados; e, por fim, as conclusões do estudo.

\section{Inovação Responsável}

Os termos "inovação responsável" (responsible innovation - RI) e "pesquisa e inovação responsáveis" (responsible research and innovation - RRI) possuem relação com desenvolvimento responsável. São consequência de discussões anteriores sobre as implicações éticas, legais e sociais da pesquisa e da integridade da pesquisa. (Owen, Macnaghte, \& Stilgoe, 2012)

O conceito mais utilizado é o proposto por von Schomberg95 (2012, p. 280) que prevê que a

Responsible Research and Innovation is a transparent, interactive process by which societal actors and innovators become mutually responsive to each other with a view on the (ethical) acceptability, sustainability and societal desirability of the innovation process and its marketable products (in order to allow a proper embedding of scientific and technological advances in our society)

Stahl (2013) propôs um novo conceito, que, segundo ele, é consistente e compreende as definições mais citadas no momento, reconhecendo, contudo, o histórico de atividades relacionadas a IR. Para Stalh $(2013$, p. 1), inovação responsável é

as a higher-level responsibility or meta-responsibility that aims to shape, maintain, develop, coordinate and align existing and novel research and innovation-related processes, actors and responsibilities with a view to ensuring desirable and acceptable research outcomes.

Além desses, outros autores propuseram conceitos para IR, como Burget, Bardone e Pedaste (2017). Wickson e Carew (2014) apontam para a diferença na terminologia, na orientação, na profundidade de descrição e na ênfase dos conceitos. No entanto, os autores 
perceberam certas características compartilhadas e que podem ser identificadas como fundamentais para o conceito emergente:

(1) Foco no atendimento de necessidades e desafios sócio-ecológicos significativos;

(2) Compromisso de envolver ativamente uma série de partes interessadas com o objetivo de uma melhor tomada de decisão e aprendizado mútuo;

(3) Tentativa dedicada de antecipar problemas potenciais, avaliar as alternativas disponíveis e refletir sobre os valores subjacentes, os pressupostos e as crenças; e

(4) Vontade entre todos os participantes de agir e se adaptar de acordo com essas ideias.

Predominantemente, os conceitos se referem a uma governança adaptativa e antecipatória de pesquisa e inovação (Owen, \& Goldberg, 2010). A governança antecipatória motiva a reflexão de cientistas, engenheiros, decisores políticos e outros públicos sobre seu papel nas novas tecnologias (Guston, 2014).

Ao considerar a inovação como um processo, a IR denota uma orientação para antecipação, inclusão, capacidade de resposta e reflexividade (Van Oudheusden, 2014). Essas quatro dimensões implicam um compromisso coletivo e contínuo de ser (Stilgoe, Owen, \& Macnaghten, 2013). A antecipação descreve e analisa os impactos - econômicos, sociais, ambientais ou outros - pretendidos e potencialmente não intencionais que possam surgir e é sustentada por metodologias que incluem a previsão, avaliação de tecnologias e desenvolvimento de cenários. A reflexão reflete sobre os propósitos subjacentes, motivações e impactos potenciais daquilo que é conhecido (incluindo as áreas de regulação, revisão ética ou outras formas de governança que podem existir) e o que não é conhecido (incertezas associadas, riscos, áreas de ignorância, pressupostos, questões e dilemas). A inclusão se refere a diminuição da autoridade de especialistas, com a inclusão de novas vozes na governança da ciência e da inovação como parte de uma busca pela legitimação. Por fim, a responsividade (capacidade de resposta) significa que a inovação responsável exige uma capacidade para mudar a forma ou direção em resposta aos valores das partes interessadas e públicos e mudanças nas circunstâncias. Esse deve ser um processo de aprendizagem adaptativo, inclusivo e aberto (capacidades dinâmicas) (Owen, Stilgoe, Macnaghten, Gorman, Fisher, \& Guston, 2013).

Duas novas perspectivas emergem nas discussões sobre IR. A sustentabilidade diz respeito a colaboração entre ciência e tecnologia como um fator chave para garantir um crescimento econômico sustentável e inclusivo. Por fim, o cuidado é a dimensão que compete ao domínio público para que os próprios cidadãos sejam responsáveis pelas decisões e ações realizadas em seu nome, através de habilidades conjuntas para perceber, agir e julgar (Burget, Bardone, \& Pedaste, 2017).

Em suma, se verifica que o conceito de inovação responsável amplia as definições tradicionais de inovação ao considerar o contexto ético, social e ambiental ao longo do processo de inovação tradicional.

\section{Metodologia}

O objetivo deste estudo é o de analisar a produção científica nacional e internacional sobre a aplicação do conceito de inovação responsável voltada à área de gestão e negócios. Para tanto foi realizado estudo bibliométrico, visando mapear a área de conhecimento do campo de Inovação Responsável, através da abordagem quantitativa e estatística de diversos dados bibliográficos (Vanti, 2002). Em julho de 2017 foi realizada pesquisa bibliográfica, de caráter exploratória, selecionando através da ferramenta Web of Science, artigos que 
continham as palavras-chave "responsible innovation" ou "responsible research and innovation", "inovação responsável" ou "pesquisa e inovação responsável" no tópico. A ferramenta Web of Science foi selecionada por ser a base com maior volume de dados disponíveis gratuitamente nas universidades brasileiras, através da agência de pesquisa de pós-graduação CAPES (Motta, \& Quintella, 2012).

A pesquisa preliminar destacou 240 artigos internacionais, de diversas áreas como engenharia, filosofia, biotecnologia, entre outras. A área de business economics destacou 33 artigos, que representou $13 \%$ dos estudos.

Como nenhum artigo nacional foi localizado, se realizou nova pesquisa no Portal de Periódicos CAPES. Na busca por assunto "inovação responsável" ou "pesquisa e inovação responsável" foram localizados seis artigos, sendo que somente um deles se enquadrou para análise. Os demais eram publicações em revistas não acadêmicas ou de origem lusitana.

Sendo assim, esse estudo considerou os 34 artigos finais, demonstrados no Apêndice A.

A etapa seguinte foi a análise estatística das publicações e posterior análise de conteúdo (Bardin, 1995). Na fase de pré-análise foi feita leitura prévia dos artigos, buscandose identificar os indicadores que norteariam a etapa seguinte. $\mathrm{Na}$ fase de exploração do material, foi realizada a definição das categorias a serem analisadas, quais sejam: foco dos estudos, lentes teóricas associadas ao conceito e pesquisa aplicada. Essas categorias foram identificadas através da leitura integral dos artigos. Por fim foi realizada a terceira fase, a de tratamento dos resultados.

\section{Estudos Sobre Inovação Responsável no Contexto de Gestão e Negócios}

A primeira pesquisa relacionada à gestão e negócios foi publicada em 2009, com o objetivo de explorar arranjos de governança para pesquisas em nanotecnologia (Robinson, 2009).

Contudo, foi a partir de 2012 que houve um incremento no número de pesquisas, conforme verificado na figura 1.

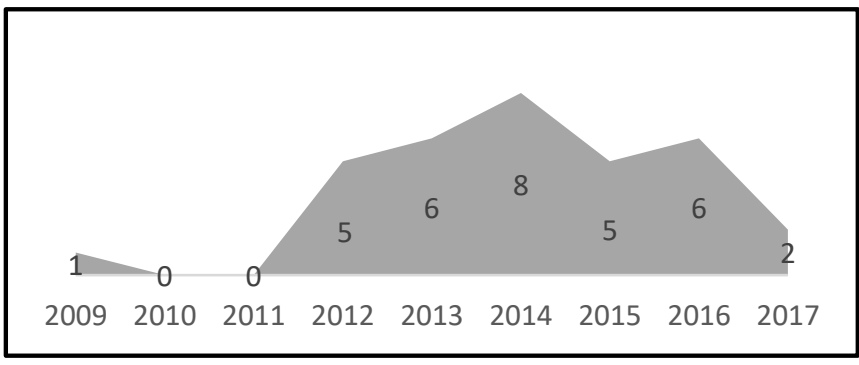

Figura 1. Número de artigos por ano

Fonte: elaborado pela autora (2017).

Esses artigos foram publicados em revistas como Technological Forecasting and Social Change, Information \& Management, Business Strategy and the Environment e Research Policy, consideradas de alto impacto. O quadro 1 apresenta a fonte das publicações e seu impacto. 
Quadro 1. Fonte das publicações

\begin{tabular}{|c|c|}
\hline PERIÓDICO & QUALIS \\
\hline Business Strategy And The Environment & Q1 \\
\hline Economy And Society & Q1 \\
\hline Food Policy & Q1 \\
\hline Futures & Q1 \\
\hline Information \& Management & Q1 \\
\hline Research Policy & Q1 \\
\hline Technological Forecasting And Social Change & Q1 \\
\hline Journal Of Business Ethics & Q2 \\
\hline Science And Public Policy & Q2 \\
\hline Journal Of Economics \& Management Strategy & Q3 \\
\hline Technology Analysis \& Strategic Management & Q3 \\
\hline International Journal Of Technology Management & Q4 \\
\hline Revista de Administração FACES Journal & $\mathrm{B} 2$ \\
\hline \multicolumn{2}{|l|}{ Journal Of Innovation Economics \& Management } \\
\hline $\begin{array}{l}\text { Proceedings Of The 11th European Conference On Innovation And } \\
\text { Entrepreneurship }\end{array}$ & \\
\hline $\begin{array}{l}\text { Proceedings Of The } 7 \text { th International Management Conference: New } \\
\text { Management For The New Economy }\end{array}$ & \\
\hline World Conference On Technology, Innovation And Entrepreneurship & \\
\hline
\end{tabular}

Fonte: elaborado pela autora (2017).

Importante destacar que do total de publicações, somente uma foi realizada no Brasil. As demais são originárias de países europeus, como Inglaterra, Países Baixos, França, Espanha, Itália, Dinamarca e Áustria, assim como Estados Unidos. Se percebe que o tema ainda não se expandiu para países em desenvolvimento.

Já a figura 2 demonstra os principais termos encontrados como palavras-chaves dos artigos. Para melhor visualização, os termos "innovation", "research" e "responsible" foram excluídos.

Se destacaram termos como development, social, science e governance, com sete ocorrências cada. Esses são seguidos dos termos nanotechnology, sustainable, corporate, responsibility, technical e technology, com cinco ocorrências cada. 


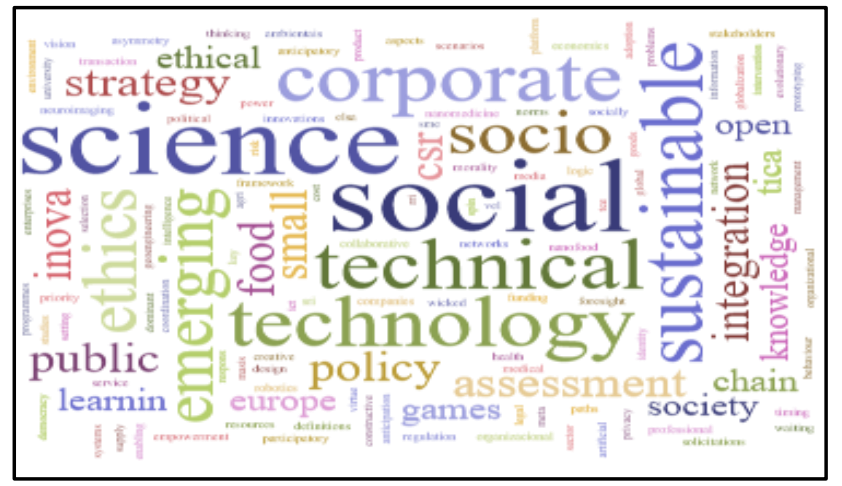

Figura 2. Nuvem de palavras-chave

Fonte: elaborado pela autora (2017)

A ainda pouca abrangência de termos, aliada ao pequeno número de pesquisas, assim como as recentes datas de publicação demonstra que ainda há um campo inexplorado nos estudos sobre inovação responsável na área de gestão e negócios.

Os estudos sobre inovação e pesquisa responsável apresentam diferentes focos de estudo, lentes teóricas associadas ao conceito e pesquisa aplicada, apresentados a seguir.

\subsection{Focos de Estudo}

A análise de conteúdo dos artigos apontou para diferentes focos de estudo, lentes teóricas, além do objeto dos estudos empíricos. Esses focos são apresentados nos subitens a seguir.

\subsubsection{Processo de Desenvolvimento da Inovação Responsável}

A metodologia responsável de gestão da inovação é desenvolvida com base em processos de gestão da inovação e métricas que dependem dos indicadores de resultados econômicos, sociais, ambientais e vinculados a estratégias (Petraite, \& Ceicyte, 2014). Os defensores da inovação responsável (IR) discutem sobre o paradigma da estagnação irresponsável, ao argumentarem que essa estagnação também pode propor uma discussão fundamentada sobre o consumo de recursos e o ritmo de desenvolvimento em setores e tecnologias excessivamente produtivas ou muito arriscadas, como parte intrínseca da inovação responsável, em vez de seu oposto (de Saille, \& Medvecky, 2016).

A inovação responsável implica um alinhamento do que os desenvolvedores e os atores da sociedade percebem como os problemas e propósitos das novas tecnologias. Com isso, o desafio é identificar prospectivamente as preocupações potenciais e barreiras (sistêmicas) que podem dificultar o desenvolvimento e a incorporação da inovação (Arentshorst, Buning, Boon, \& Broerse, 2015).

Como processo de criação da inovação responsável, uma abordagem pioneira baseada em métodos de design centrados no usuário, o design thinking representa uma combinação única de rigor científico e técnico para resolver problemas complexos, como a integração operacional de responsabilidade ao longo do processo de inovação. É necessária orientação na direção que aborda responsabilidade e imperativos de desempenho, sendo necessário adaptar o processo de inovação responsável ao setor e à empresa. Esta fase de adaptação é parte integrante do método de design thinking, permitindo que a equipe multidisciplinar defina as condições específicas para a incerteza em seu setor específico (Pavie, \& Carthy, 2015). 


\subsection{2 Âmbito da inovação}

Dentro do contexto da inovação responsável, Owen et al. (2012) propõe que a ciência seja feita para e com a sociedade. No que diz respeito sobre a ciência para a sociedade, a preocupação é com os propósitos da ciência e inovação, e as motivações e intenções subjacentes para estas. Já no que se refere a ciência com a sociedade, há ênfase na integração e institucionalização de mecanismos estabelecidos de reflexão, antecipação e deliberação inclusiva em torno dos processos de pesquisa e inovação. Nesses contextos, os autores propõem a reestruturação da responsabilidade, ou seja, novas responsabilidades, não só aos cientistas, universidades, inovadores e empresas, mas também aos decisores políticos e financiadores de pesquisa.

A amplitude de envolvidos é proposta através de uma estrutura para a tomada de decisões estratégicas por empresas incorporadas em sistemas de inovação em quádrupla hélice. Essa proposta aborda as empresas inovadoras que podem incorporar de forma funcional e orgânica processos de deliberações mais amplos associados à pesquisa e inovação responsáveis envolvendo atores dos setores público e privado, bem como organizações da sociedade civil ao longo dos preceitos da estrutura de inovação quádrupla-hélice (ParedesFrigolett, 2016).

O espaço onde a IR é aplicado é apresentado graficamente por Stahl (2013).
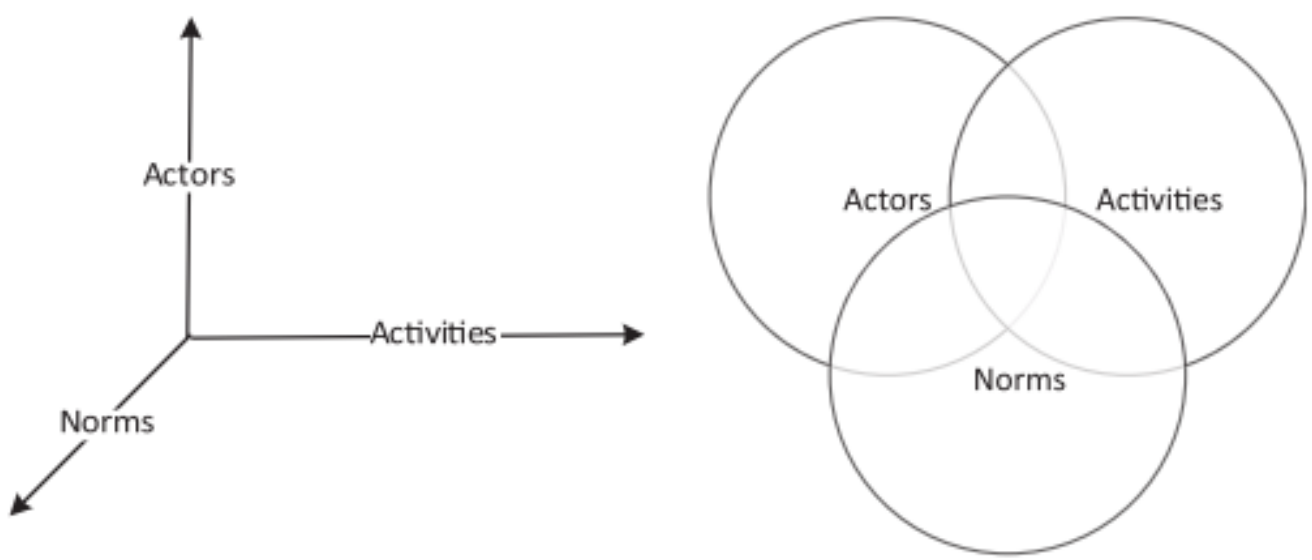

Figura 3. Tentativas diferentes para representar graficamente o espaço do IR. Fonte: Stahl (2013, p. 3).

O estudo não aprofunda o aspecto das atividades, mas destaca pesquisa prospectiva, o envolvimento das partes interessadas, a integração de valores éticos, as estruturas de responsabilidade como códigos de ética ou códigos de conduta. Os atores incluem pesquisadores individuais, organizações de pesquisa (tanto públicos como privados), comitês de ética de pesquisa e seus membros, usuários de pesquisa e inovação, atores da sociedade civil, decisores políticos em diferentes níveis, órgãos profissionais, legisladores, organizações educacionais (por exemplo, escolas e universidades) e organismos públicos (das autoridades locais às estruturas regionais). E no que se refere as normas, Stahl (2013) sugere a inclusão de normas e os princípios da governança democrática existentes, como os tratados europeus, princípios estabelecidos pela ONU, Convenção Européia de Direitos Humanos e pela Carta 
Européia dos Direitos Fundamentais, assim como o Projeto de Código de Ética da UNESCO para a Sociedade da Informação.

\subsubsection{Relação com Stakeholders}

A participação de todas as partes interessadas no processo de desenvolvimento de novos produtos (NPD) é tratado em estudos como o de Ratiu e Mortan (2013), SchaperRinkel (2013), Yenicioglu e Suerdem (2015) e Ceccaldi (2016). Em comum, os autores trazem a necessidade de inclusão de diversos atores no contexto da inovação responsável.

Para Yenicioglu e Suerdem (2015), é uma questão importante para a democratização do uso de bens e serviços e a promoção da pesquisa e inovação responsáveis. Os autores consideram complexa e difusa a organização e coordenação dos papéis baseados na participação, por conta do viés político, no sentido de persuadir outras pessoas a alguma forma de ação. Essa coordenação é destacada também por Ceccaldi (2016), no contexto de micro e pequenas empresas, ao buscar compreender o papel que os empresários desempenham em cada uma das fases de desenvolvimento da IR. Para o autor, cada decisão e cada ato de líder tem um impacto sobre como uma organização evolui.

Ainda o impacto que as decisões das empresas possuem sobre a comunidade é tratado por Ratiu e Mortan (2013), ao reforçar que a gestão das empresas e a forma como entendem a responsabilidade social podem ter um impacto negativo ou positivo nos consumidores, parceiros de negócios, comunidades e meio ambiente.

E para estreitar essas relações, novos modelos são apresentados. A análise de tecnologia orientada para o futuro (FTA) tem sido usada para criar conceitos visionários e para promover a cooperação entre agências, departamentos do governo federal, academia e partes interessadas (Schaper-Rinkel, 2013).

\subsubsection{Políticas Públicas}

Diversos estudos trazem o papel das políticas governamentais como agentes de inovação responsável (Mejlgaard, \& Bloch, 2012; Rodríguez, Fisher, \& Schuurbiers, 2013; Fisher, \& Maricle, 2013). Se destaca o estudo de Rodríguez et al., 2013) que dá ênfase na integração sócio-técnica. Os autores identificaram três características-chave da política europeia de pesquisa de integração sócio-técnica, argumentando que o discurso político no nível de definição da agenda, ao longo dos anos, pediu que a integração seja mais generalizada (aproximando-se das práticas reais de P\&D), extensiva (abordando um alcance mais amplo de áreas de pesquisa) e diversas (envolvendo uma gama mais ampla de tipos de integração).

\subsection{5 Ética e poder}

A ética está atrelada diretamente ao conceito de inovação responsável. E essa relação é verificada em estudos como o de Pandza e Ellwood (2013), que sugere a interação entre a estratégia das instituições e ética, uma vez que é incipiente o processo de institucionalização da inovação responsável.

Já Stahl, Eden, Jirotka, \& Coeckelbergh (2014a) relacionam ética e tecnologias futuras. Os autores sugerem a conexão de convicções morais com o conhecimento acadêmico de possíveis futuros. Salientam que questões éticas estão no cerne da condição humana e a 
maioria das pessoas tem fortes convicções morais. Sugerem que essa é uma condição importante para mudanças bem-sucedidas.

A relação entre ética e inovação sustentável é abordada por Aloise (2017). O autor considera que fatores limitantes ao desenvolvimento sustentável estão na cultura organizacional, na lógica puramente mercantil que os mercados exigem e na falta de sensibilidade ética dos empresários.

Já o poder é abordado por de Bakker, de Lauwere, Hoes, \& Beekman (2014). Para os autores, o poder pode se referir às relações entre as diferentes partes interessadas e aos recursos que eles têm para controlar ou influenciar (a política em) inovação.

\subsection{Lentes teóricas}

Muitos dos artigos sobre inovação responsável utilizam exclusivamente o tema IR como teoria. Alguns, contudo, o fazem analisando sob uma lente teórica, conforme apresentado a seguir.

\subsubsection{Governança}

De forma mais ampla, o artigo de Voegtlin e Scherer (2017) argumenta que a inovação responsável contribui para o desenvolvimento sustentável e consiste em três dimensões. A primeira é a de que as inovações evitam prejudicar as pessoas e o planeta. A segunda é a de que as inovações "fazem bem" oferecendo novos produtos, serviços ou tecnologias que promovem o desenvolvimento sustentável. E a terceira é a de que esquemas de governança global facilitam inovações que evitem danos e "fazem o bem". O artigo discute os esquemas de governança global baseados em deliberações como meio de promover essa inovação responsável.

Outros estudos tratam de aspectos gerais da governança como cenários co-evolutivos (Robinson, 2009), quadros regulatórios do governo, voluntárias - sociedade civil e coregulação (D'Silva et al., 2012), na capacidade de resposta na governança de RRI (Nielsen, 2016) e na relação entre governança e fatores cognitivos (Nielsen, 2016). Nesse caso, o conceito articula a necessidade de intercâmbio mútuo pelo qual os atores sociais se tornam receptivos uns aos outros no início da inovação, com o objetivo de facilitar a inovação eticamente aceitável e sustentável.

No campo da nanotecnologia, a governança tem papel fundamental. Mas apesar dos repetidos pedidos de novas formas de inovação e governança, incluindo inovação responsável, governança antecipada e governança orientada para a sustentabilidade, a nanotecnologia continua a ser principalmente inovada na sequência de esquemas convencionais - com falhas persistentes e impactos negativos (Wiek, Foley, Guston, \& Bernstein, 2016).

Contudo, a principal referência sobre governança em inovação responsável é o trabalho de Stilgoe, Owen e Macnaghten (2013). Nele, os autores apresentam quatro dimensões da inovação responsável, que são:

1) A antecipação envolve o pensamento sistemático com o objetivo de aumentar a resiliência, ao mesmo tempo que revela novas oportunidades de inovação e a elaboração de agendas para pesquisas de risco socialmente robustas.

2) A reflexão, ao nível da prática institucional, significa realizar um espelho para as próprias atividades, compromissos e premissas, estar ciente dos limites do conhecimento e ter consciência de que um determinado enquadramento de uma questão pode não ser universalmente realizado. 
3) A inclusão - a diminuição da autoridade de especialistas, a formulação de políticas de cima para baixo tem sido associada a um aumento da inclusão de novas vozes na governança da ciência e da inovação como parte de uma busca pela legitimação;

4) A responsividade (capacidade de resposta) - a inovação responsável exige uma capacidade para mudar a forma ou direção em resposta aos valores das partes interessadas e públicos e mudanças nas circunstâncias.

Esse framework busca responder a uma lacuna de legitimidade no envolvimento deliberativo em questões de ciência e tecnologia, seja na forma como os processos são criados e executados, seja na legitimidade da produção, seja na eficácia da governança.

\subsubsection{Recursos}

A resource-based view é utilizada como base para estudo em micro e pequenas empresas (Halme, \& Korpela, 2014). Nesse contexto, as inovações responsáveis podem ser criadas com combinações de recursos muito diferentes, tanto em termos de quantidade quanto de qualidade dos recursos. Os autores destacam que pequena e média empresas ainda têm que lidar com a escassez de recursos.

\subsubsection{Custos de Transação}

Pyszka (2012) dedicou o artigo a olhar para as inovações sob a perspectiva dos Custos de Transação (CT) e da Responsabilidade Social Corporativa. No que diz respeito a CT, buscou demonstrar que independentemente do tipo de regulamento de transações, a empresa deve levar em consideração o ambiente regulatório associado ex ante e ex post na administração da transação, para moldar conscientemente o contexto social e a justiça interacional.

Devido a relevância da responsabilidade social corporativa no contexto de inovação responsável, o tema será abordado separadamente, a seguir.

\subsubsection{Responsabilidade Social Corporativa (RSC)}

A RSC e sua relação com a inovação são abordados em alguns estudos. Sua ligação é apresentada por Spena e de Chiara (2012), através de três aspectos principais:

1) Novas inovações de produtos / serviços - que se refere ao aumento de novas oportunidades de negócios ligadas a novos problemas sociais e ambientais;

2) Novos processos de inovação - que se refere a uma forma mais eficaz de criar e entregar produtos e serviços através de práticas de RSE consistentes; e

3) Inovação organizacional, que se refere a novas formas de gerenciar recursos e atividades internas e externas de acordo com uma perspectiva de RSE mais integrada.

Os autores ainda propõe que a CSR pode contribuir para a inovação e boas práticas de negócios e desempenho através da identificação de oportunidades de negócios que exploram novos caminhos que atendem aos desafios ambientais e da sociedade, através da criação de um local de trabalho abrangente na cadeia de suprimentos responsivo e comprometido com questões sociais e ambientais e através da criatividade que alavanca práticas sociais (como diversidade e inclusão), promovendo um conjunto comum de valores.

Pyszka (2012) apresenta o conceito de Inovações Socialmente Responsáveis (SRI), que são sensíveis às necessidades sociais combinadas com atividades empresariais importantes ou construir o conhecimento e a experiência da empresa, cientes das 
conseqüências que podem resultar dessas inovações, ambas usadas no âmbito de produtos e serviços legítimos (públicos e militares) e ilegais (terrorismo, pirataria, etc.). As inovações socialmente responsáveis parecem ser uma oportunidade para criar melhores inovações com menores custos de implementação, também com base nas novas formas de cooperação com diferentes redes (alianças, cluster, virtual, etc.).

E sua relação com empresas agroalimentares, uma vez que suas ações impactam diretamente consumidores, parceiros de negócios, comunidades e meio ambiente (Ratiu, \& Mortan, 2013).

\subsection{Pesquisa aplicada}

Muitos dos estudos sobre IR são aplicados a áreas ou setores específicos. Destacam-se estudos em saúde e alimentos (Ratiu, \& Mortan, 2013; Khan, Timotijevic, Newton, Coutinho, Llerena, Ortega, \& Urban, 2016), adoção de tecnologia verde (Galasso, \& Tombak, 2014), aplicado a pequenas empresas (Ceccaldi, 2016), sistemas de informação (Stahl et al., 2014a; Stahl et al., 2014b) e superinteligência (Brundage, 2015).

Mas a maioria dos estudos aplicados se referem a nanotecnologia (de Bakker et al., 2014; Foley, \& Wiek, 2014; Fisher, \& Maricle, 2014; Wiek et al., 2016). Enquanto tratam da integração e capacidade de resposta ao nível da prioridade de pesquisa (Fisher, \& Maricle, 2014), a governança na nanotecnologia é percebida exigindo mudanças difíceis em todas as etapas e atores no processo de inovação Wiek et al., 2016). Dentro dessa ótica, Foley e Wiek (2014) percebem quatro cenários para o setor, quais sejam a inovação em conformidade com o mercado, a inovação através do empreendedorismo social, a inovação através de colaboração fechada e a inovação de código aberto.

O ambiente universitário é tratado através das spin-offs universitárias (van Geenhuizen, \& Ye, 2014). A análise se concentra em jovens empresas spin-off universitárias como um canal importante para trazer inovações responsáveis da universidade para o mercado enquanto se conecta com níveis de redes que suportam mudanças do sistema sócio-técnico, influenciadas pelo foco (mercado de produtos) e a seletividade na escolha de parceiros ligados ao capital profissional, ao acesso ao mercado, à credibilidade e aos ativos complementares. Já Savoia, Lefebvre, Millot e Bocquet (2017) apresentam o conceito de lojas científicas, que são interfaces ciência-sociedade que seguem uma abordagem ascendente, ao trabalhar na tradução de demandas de pesquisa de base social em termos de questões científicas.

\section{Conclusão}

Ao se analisar a aplicação do conceito de inovação responsável, se verificou que esse é um tema relativamente recente na área de gestão e negócios. Os principais estudos abordam a IR sob a ótica da governança (Stilgoe et al.., 2013) e das políticas públicas (Owen et al.., 2012). Outros estudos seguem os mesmos temas, como Robinson (2009), Mejlgaard \& Bloch (2012), D'Silva et al. (2012), Rodríguez et al. (2013), Fisher \& Maricle (2013), Nielsen (2016), Wiek et al. (2016) e Voegtlin e Scherer (2017).

Atrelado ao aspecto da governança, destaca-se aqui as teorias de base utilizadas nas pesquisas. Muitos estudos utilizam somente o conceito de IR, sem atrela-lo a alguma lente teórica. Alguns estudos, contudo, associam IR as teorias de Recursos, Custos de Transação, Stakeholders e Responsabilidade Social Corporativa. Aponta-se aqui a possibilidade de estudos futuros associando a outras teorias, como Teoria Institucional, que pode colaborar no entendimento do papel de alguns atores, assim como, aprofundar a teoria dos recursos, não 
ficando exclusiva à RBV mas também a capacidades dinâmicas, que pode auxiliar na resposta de como se dá o processo da IR no contexto organizacional.

Os estudos de IR também foram aplicados em poucas áreas, tais como saúde e alimentos (Ratiu \& Mortan, 2013; Khan et al., 2016), adoção de tecnologia verde (Galasso \& Tombak, 2014), aplicado a pequenas empresas (Ceccaldi, 2016), sistemas de informação (Stahl et al., 2014), superinteligência (Brundage, 2015) e nanotecnologia (de Bakker et al., 2014; Foley, \& Wiek, 2014; Fisher, \& Maricle, 2014; Wiek et al., 2016).

E apesar de as interações entre universidade e empresa serem consideradas como condição importante para a inovação (Khan et al. 2016), o papel da universidade como ator no processo de IR é pouco explorado. Destacam-se somente os estudos de van Geenhuizen \& Ye (2014) e Savoia et al. (2017). Aqui se percebe outra linha de pesquisa que pode ser realizada, ao avaliar de que forma a universidade influencia a responsabilidade na inovação daquelas empresas com que se relaciona.

Uma vez que somente uma base de dados (Web of Science) foi utilizada para analisar o conceito de IR no âmbito de gestão e negócios, sugere-se novas pesquisas ampliando a utilização de novas bases. Também se sugere a ampliação da análise do conceito, não somente vinculado à gestão e negócios, mas a outras áreas do conhecimento.

\section{REFERÊNCIAS}

Aloise, P. G. (2017). Relações entre ética organizacional, inovações ambientais e sustentabilidade. Revista de Administração FACES Journal, 16(2).

Arentshorst, M. E., de Cock Buning, T., Boon, W. P., \& Broerse, J. E. (2015). Prospecting responsible technology paths: Management options for an appropriate societal embedding of medical neuroimaging. Science and Public Policy, 42(6), 775-788.

Bardin, L. (1995). Análise de conteúdo. Lisboa: Edições 70.

Burget, M., Bardone, E., \& Pedaste, M. (2017). Definitions and Conceptual Dimensions of Responsible Research and Innovation: A Literature Review. Science and engineering ethics, 23(1), 1-19.

Brundage, M. (2015). Taking superintelligence seriously: Superintelligence: Paths, dangers, strategies by Nick Bostrom (Oxford University Press, 2014). Futures, 72, 32-35.

Ceccaldi, V. (2016). How Responsible Innovation Strategies Emerge in Very Small Enterprises: The Case of a Small Wine-Growers' Cooperative. In Proceedings of The 11th European Conference on Innovation and Entrepreneurship 15-16 September 2016 (p. 116).

Comissão Europeia (2002) The Sixth Framework Programme. Disponível em: < https://ec.europa.eu/research/fp6/index_en.cfm>

(2013) The Seventh Framework Programme. Disponível em: < https://ec.europa.eu/research/fp7/index_en.cfm>

de Bakker, E., de Lauwere, C., Hoes, A. C., \& Beekman, V. (2014). Responsible research and innovation in miniature: Information asymmetries hindering a more inclusive 'nanofood'development. Science and Public Policy, 41(3), 294-305. 
de Oslo, M. (1997). Manual de Oslo. Recuperado de http://gestiona. com. br/wpcontent/uploads/2013/06/Manual-de-OSLO-2005. pdf.

de Saille, S., \& Medvecky, F. (2016). Innovation for a steady state: a case for responsible stagnation. Economy and Society, 45(1), 1-23.

D'Silva, J., Robinson, D. K., \& Shelley-Egan, C. (2012). A game with rules in the makinghow the high probability of waiting games in nanomedicine is being mitigated through distributed regulation and responsible innovation. Technology Analysis \& Strategic Management, 24(6), 583-602.

European Commission, 2013. The EU Framework Programme for Research and Innovation [WWW Document], URL <http://ec.europa.eu/research/ horizon2020/index_en.cfm?pg=h2020>.

Fisher, E., \& Maricle, G. (2014). Higher-level responsiveness? Socio-technical integration within US and UK nanotechnology research priority setting. Science and Public Policy, 42(1), $72-85$.

Foley, R. W., \& Wiek, A. (2014). Scenarios of nanotechnology innovation vis-à-vis sustainability challenges. Futures, 64, 1-14.

Galasso, A., \& Tombak, M. (2014). Switching to Green: The Timing of Socially Responsible Innovation. Journal of Economics \& Management Strategy, 23(3), 669-691.

Halme, M., \& Korpela, M. (2014). Responsible Innovation toward sustainable development in small and medium-sized enterprises: a resource perspective. Business Strategy and the Environment, 23(8), 547-566.

Khan, S. S., Timotijevic, L., Newton, R., Coutinho, D., Llerena, J. L., Ortega, S., ... \& Urban, C. (2016). The framing of innovation among European research funding actors: Assessing the potential for 'responsible research and innovation'in the food and health domain. Food Policy, 62, 78-87.

Mejlgaard, N., \& Bloch, C. (2012). Science in society in Europe. Science and Public Policy, 39(6), 695-700.

Nações Unidas (2015). Transforming our world: the 2030 Agenda for Sustainable Development. General Assembly. Seventieth session Agenda items 15 and 116.

Nielsen, M. V. (2016). The concept of responsiveness in the governance of research and innovation. Science and Public Policy, 43(6), 831-839.

Owen, R., Macnaghten, P., \& Stilgoe, J. (2012). Responsible research and innovation: From science in society to science for society, with society. Science and Public Policy, 39(6), 751760 .

Pandza, K., \& Ellwood, P. (2013). Strategic and ethical foundations for responsible innovation. Research Policy, 42(5), 1112-1125. 
Paredes-Frigolett, H. (2016). Modeling the effect of responsible research and innovation in quadruple helix innovation systems. Technological Forecasting and Social Change, 110, 126133.

Pavie, X., \& Carthy, D. (2015). Leveraging uncertainty: a practical approach to the integration of responsible innovation through design thinking. Procedia-Social and Behavioral Sciences, 213, 1040-1049.

Petraitè, M., \& Čeičytè, J. (2014). Conceptual Model for Responsible Innovation Management in Business Organizations. Procedia-Social and Behavioral Sciences, 156, 121124.

Pyszka, A. (2012). Socially Responsible Innovations (SRI) from a Transactional Cost Perspective-A Chance for Growth and Development or a Waste of Time and Money?. Business and non-profit organizations facing increased competition and growing customers'demands, 18, 57.

Ratiu, P., \& Mortan, M. (2013). Ethical Aspects Of Decision-Making In Agri-Food Enterprises.

Robinson, D. K. (2009). Co-evolutionary scenarios: An application to prospecting futures of the responsible development of nanotechnology. Technological Forecasting and Social Change, 76(9), 1222-1239.

Rodríguez, H., Fisher, E., \& Schuurbiers, D. (2013). Integrating science and society in European Framework Programmes: Trends in project-level solicitations. Research Policy, 42(5), 1126-1137.

Spena, T.R, \& De Chiara, A. (2012). CSR, innovation strategy and supply chain management: toward an integrated perspective. International Journal of Technology Management, 58(1/2), 83-108.

Savoia, A., Lefebvre, B., Millot, G., \& Bocquet, B. (2017). The Science Shop Concept and its Implementation in a French University. Journal of Innovation Economics \& Management, (1), 97-117.

Schaper-Rinkel, P. (2013). The role of future-oriented technology analysis in the governance of emerging technologies: The example of nanotechnology. Technological Forecasting and Social Change, 80(3), 444-452.

Scherer, A. G., Palazzo, G., \& Baumann, D. (2006). Global rules and private actors: Toward a new role of the transnational corporation in global governance. Business Ethics Quarterly, 16(4), 505-532.

Stahl, B. C. (2013). Responsible research and innovation: The role of privacy in an emerging framework. Science and Public Policy, 40(6), 708-716. 
, Eden, G., Jirotka, M., \& Coeckelbergh, M. (2014a). From computer ethics to responsible research and innovation in ICT: The transition of reference discourses informing ethics-related research in information systems. Information \& Management, 51(6), 810-818.

, McBride, N., Wakunuma, K., \& Flick, C. (2014b). The empathic care robot: A prototype of responsible research and innovation. Technological Forecasting and Social Change, 84, 74-85.

Stilgoe, J., Owen, R., \& Macnaghten, P. (2013). Developing a framework for responsible innovation. Research Policy, 42(9), 1568-1580.

van Geenhuizen, M., \& Ye, Q. (2014). Responsible innovators: open networks on the way to sustainability transitions. Technological Forecasting and Social Change, 87, 28-40.

Vanti, N. Da bibliometria à webometria: uma exploração conceitual dos mecanismos utilizados para medir o registro da informação e a difusão do conhecimento. Ciência da Informação, v. 31, n. 2, p. 152-162, maio/ ago. 2002.

Voegtlin, C., \& Scherer, A. G. (2017). Responsible innovation and the innovation of responsibility: Governing sustainable development in a globalized world. Journal of Business Ethics, 143(2), 227-243.

von Schomberg95, R. (2012). The quest for the "right" impacts of science and technology. An outlook towards a framework for responsible research and innovation. Les nanotechnologies: vers un changement d'échelle éthique?, 269.

Wiek, A., Foley, R. W., Guston, D. H., \& Bernstein, M. J. (2016). Broken promises and breaking ground for responsible innovation-intervention research to transform business-asusual in nanotechnology innovation. Technology Analysis \& Strategic Management, 28(6), 639-650.

Yenicioglu, B., \& Suerdem, A. (2015). Participatory new product development-a framework for deliberately collaborative and continuous innovation design. Procedia-Social and Behavioral Sciences, 195, 1443-1452. 


\section{Apêndice A - Artigos selecionados}

\begin{tabular}{|c|c|c|}
\hline TÍTULO & AUTORES & ANO \\
\hline $\begin{array}{l}\text { Co-evolutionary scenarios: An application to prospecting } \\
\text { futures of the responsible development of nanotechnology }\end{array}$ & Robinson, DKR & 2009 \\
\hline $\begin{array}{l}\text { A game with rules in the making - how the high probability } \\
\text { of waiting games in nanomedicine is being mitigated } \\
\text { through distributed regulation and responsible innovation }\end{array}$ & $\begin{array}{l}\text { D'Silva, J; Robinson, DKR; Shelley- } \\
\text { Egan, C }\end{array}$ & 2012 \\
\hline $\begin{array}{l}\text { CSR, innovation strategy and supply chain management: } \\
\text { toward an integrated perspective }\end{array}$ & Spena, TR; de Chiara, A & 2012 \\
\hline $\begin{array}{l}\text { Responsible research and innovation: From science in } \\
\text { society to science for society, with society }\end{array}$ & Owen, R; Macnaghten, P; Stilgoe, J & 2012 \\
\hline Science in Society in Europe & Mejlgaard, N; Bloch, C & 2012 \\
\hline $\begin{array}{l}\text { Socially responsible innovations (sri) from a transactional } \\
\text { cost perspective - a chance for growth and development or } \\
\text { a waste of time and money? }\end{array}$ & Pyszka, A & 2012 \\
\hline Developing a framework for responsible innovation & Stilgoe, J; Owen, R; Macnaghten, P & 2013 \\
\hline Ethical aspects of decision-making in agri-food enterprises & Ratiu, P; Mortan, M & 2013 \\
\hline $\begin{array}{l}\text { Integrating science and society in European Framework } \\
\text { Programmes: Trends in project-level solicitations }\end{array}$ & Rodríguez, H; Fisher, E; Schuurbiers, D & 2013 \\
\hline $\begin{array}{l}\text { Responsible research and innovation: The role of privacy } \\
\text { in an emerging framework }\end{array}$ & Stahl, BC & 2013 \\
\hline $\begin{array}{l}\text { Strategic and ethical foundations for responsible } \\
\text { innovation }\end{array}$ & Pandza, K; Ellwood, P & 2013 \\
\hline $\begin{array}{l}\text { The role of future-oriented technology analysis in the } \\
\text { governance of emerging technologies: The example of } \\
\text { nanotechnology }\end{array}$ & Schaper-Rinkel, P & 2013 \\
\hline $\begin{array}{l}\text { Conceptual model for responsible innovation management } \\
\text { in business organizations }\end{array}$ & Petraite, M; Ceicyte, J & 2014 \\
\hline $\begin{array}{l}\text { From computer ethics to responsible research and } \\
\text { innovation in ICT The transition of reference discourses } \\
\text { informing ethics-related research in information systems }\end{array}$ & $\begin{array}{l}\text { Stahl, BC; Eden, G; Jirotka, M; } \\
\text { Coeckelbergh, M }\end{array}$ & 2014 \\
\hline $\begin{array}{l}\text { Responsible Innovation Toward Sustainable Development } \\
\text { in Small and Medium-Sized Enterprises: a Resource } \\
\text { Perspective }\end{array}$ & Halme, M; Korpela, M & 2014 \\
\hline $\begin{array}{l}\text { Responsible innovators: open networks on the way to } \\
\text { sustainability transitions }\end{array}$ & van Geenhuizen, M; Ye, Q & 2014 \\
\hline $\begin{array}{l}\text { Responsible research and innovation in miniature: } \\
\text { Information asymmetries hindering a more inclusive } \\
\text { 'nanofood' development }\end{array}$ & $\begin{array}{l}\text { de Bakker, E; de Lauwere, C; Hoes, } \\
\text { AC; Beekman, V }\end{array}$ & 2014 \\
\hline
\end{tabular}




\begin{tabular}{|c|c|c|}
\hline \begin{tabular}{|l} 
Scenarios of nanotechnology innovation \\
sustainability challenges
\end{tabular} & Foley, RW; Wiek, A & 2014 \\
\hline $\begin{array}{l}\text { Switching to green: the timing of socially responsible } \\
\text { innovation }\end{array}$ & Galasso, A; Tombak, M & 2014 \\
\hline $\begin{array}{l}\text { The empathic care robot: A prototype of responsible } \\
\text { research and innovation }\end{array}$ & $\begin{array}{l}\text { Stahl, BC; McBride, N; Wakunuma, K; } \\
\text { Flick, C }\end{array}$ & 2014 \\
\hline $\begin{array}{l}\text { Higher-level responsiveness? Socio-technical integration } \\
\text { within US and UK nanotechnology research priority setting }\end{array}$ & Fisher, E; Maricle, G & 2015 \\
\hline $\begin{array}{l}\text { Leveraging uncertainty: a practical approach to the } \\
\text { integration of responsible innovation through design } \\
\text { thinking }\end{array}$ & Pavie, X; Carthy, D & 2015 \\
\hline $\begin{array}{l}\text { Participatory New Product Development - A Framework } \\
\text { for Deliberately Collaborative and Continuous Innovation } \\
\text { Design }\end{array}$ & Yenicioglu, B; Suerdem, A & 2015 \\
\hline $\begin{array}{l}\text { Prospecting responsible technology paths: Management } \\
\text { options for an appropriate societal embedding of medical } \\
\text { neuroimaging' }\end{array}$ & $\begin{array}{l}\text { Arentshorst, ME; Buning, TD; Boon, } \\
\text { WPC; Broerse, JEW }\end{array}$ & 2015 \\
\hline 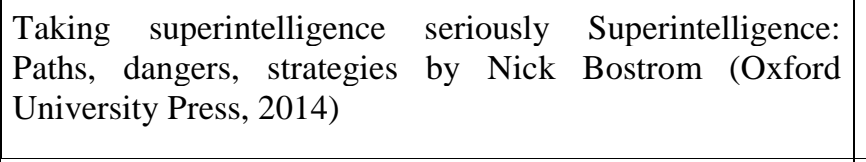 & Brundage, $\mathrm{M}$ & 2015 \\
\hline $\begin{array}{l}\text { Broken promises and breaking ground for responsible } \\
\text { innovation - intervention research to transform business-as- } \\
\text { usual in nanotechnology innovation }\end{array}$ & $\begin{array}{l}\text { Wiek, A; Foley, RW; Guston, DH; } \\
\text { Bernstein, MJ }\end{array}$ & 2016 \\
\hline $\begin{array}{l}\text { How Responsible Innovation Strategies Emerge in Very } \\
\text { Small Enterprises: The Case of a Small Wine-Growers' } \\
\text { Cooperative }\end{array}$ & Ceccaldi, V & 2016 \\
\hline $\begin{array}{l}\text { Innovation for a steady state: a case for responsible } \\
\text { stagnation }\end{array}$ & de Saille, S; Medvecky, F & 2016 \\
\hline $\begin{array}{l}\text { Modeling the effect of responsible research and innovation } \\
\text { in quadruple helix innovation systems }\end{array}$ & Paredes-Frigolett, $\mathrm{H}$ & 2016 \\
\hline $\begin{array}{l}\text { The concept of responsiveness in the governance of } \\
\text { research and innovation }\end{array}$ & Nielsen, MV & 2016 \\
\hline $\begin{array}{l}\text { The framing of innovation among European research } \\
\text { funding actors: Assessing the potential for 'responsible } \\
\text { research and innovation' in the food and health domain }\end{array}$ & $\begin{array}{l}\text { Khan, SS; Timotijevic, L; Newton, R; } \\
\text { Coutinho, D; Llerena, JL; Ortega, S; } \\
\text { Benighaus, L; ... Hadwiger, K }\end{array}$ & 2016 \\
\hline $\begin{array}{l}\text { Responsible Innovation and the Innovation of } \\
\text { Responsibility: Governing Sustainable Development in a } \\
\text { Globalized World }\end{array}$ & Voegtlin, C; Scherer, AG & 2017 \\
\hline $\begin{array}{l}\text { The science shop concept and its implementation in a } \\
\text { french university }\end{array}$ & $\begin{array}{l}\text { Savoia, A; Lefebvre, B; Millot, G; } \\
\text { Bocquet, B }\end{array}$ & 2017 \\
\hline $\begin{array}{l}\text { Relações entre ética organizacional, inovações ambientais } \\
\text { e sustentabilidade }\end{array}$ & Aloise, P. G & 2017 \\
\hline
\end{tabular}

